\title{
Combined Central Retinal Vein and Cilioretinal Artery Occlusion in Pregnancy treated with Intravitreal Triamcinolone Acetonide
}

\section{Minakshi Sheokand ${ }^{*}$, Dhir Pushkar², Bajaj Piyush ${ }^{2}$ and Dhir Parikshit $^{2}$}

${ }^{1}$ Charak Pallika Hospital, NDMC, Moti Bagh, New Delhi, India

${ }^{2}$ Dhir Hospital and Eye Institute, Meham Chowk, Bhiwani, Haryana, India

*Corresponding Author: Minakshi Sheokand, Cataract and Cornea Refractive

Services, Charak Pallika Hospital, NDMC, Moti Bagh, New Delhi, India.
Received: May 22, 2021

Published: August 06, 2021

(C) All rights are reserved by Manoj Kumar

Ghosal., et al.

\begin{abstract}
Pregnancy represents hypercoagulable state with increased risk for thrombotic events. There are few reports of pregnancy-related retinal vein occlusion cases. We report a case of 7 month pregnant female presented with a loss of vision in her right eye with prior history of pre-eclampsia in her first pregnancy. Vision in the affected eye was 6/24. Complete ophthalmic examination revealed combined central retinal vein and cilioretinal artery occlusion. Optical coherence tomography, low dose angiography and carotid doppler study was done along with complete thrombophilic screening. She was treated with anterior chamber paracentesis and topical e/d dorzolamide. After detailed counseling and an informed consent, intravitreal triamcinolone was given. Six weeks post intervention there was complete resolution of edema with best corrected visual acuity (BCVA) of 6/9. No adverse outcomes for either mother or fetus were noted. We report this case to highlight pregnancy as risk factor for combined vascular occlusion and role of intra-vitreal steroid in treatment.
\end{abstract}

Keywords: Pregnancy; Combined Vascular Occlusion; Intravitreal; Triamcinolone Acetonide

\section{Abbreviations}

CRVO: Central Retinal Vein Occlusion; CLRAO: Cilioretinal Artery Occlusion; R/E: Right Eye; L/E: Left Eye; BCVA: Best Corrected Visual Acuity; RAPD: Relative Afferent Pupillary Defect; IOP: Intraocular Pressure; OCT: Optical Coherence Tomography; Anti-VEGF: Anti Vascular Endothelial Growth Factor

\section{Introduction}

In literature, the association of pregnancy and thrombophilia has been consistently reported [1]. Pregnancy induces a hypercoagulable state and predisposes for thrombotic occlusion of vasculature in the body [1]. Various cases of retinal vascular occlusion in pregnant females has been reported [1-4]. However these cases are either retinal vein $[1,4]$ or retinal artery $[2,3]$ occlusion. We are reporting a case of combined central retinal vein occlusion (CRVO) and cilioretinal artery occlusion (CLRAO) in a pregnant female treated with intravitreal triamcinolone acetonide.

\section{Case Report}

A 26 years old Gravida 2 Para $1+0$ at 28 weeks of period of amenorrhea, complained of sudden painless diminution of vision in right eye (R/E) for 1 week duration. There is a positive history of pre-eclampsia in her previous pregnancy 3 years back. It was managed conservatively and she delivered a healthy baby at full term. 
There was no history of any problem in the present pregnancy so far. Her family history was not significant with only a positive history of hypertension in her mother.

At presentation, the best corrected visual acuity (BCVA) in R/E was 6/24 (-5.0 DS) and 6/6 in left eye (L/E) ( - 5.50 DS ). On examination, $\mathrm{L} / \mathrm{E}$ was within normal limits. $\mathrm{R} / \mathrm{E}$ showed positive relative afferent pupillary defect (RAPD). The intraocular pressure (IOP) was $16 \mathrm{mmHg}$ in R/E and $19 \mathrm{mmHg}$ in L/E. On gonioscopy, angles were open upto grade 4 according to Shaffer grading in all quadrants in both eyes. Dilated fundus examination of right eye revealed dilated, tortuous retinal veins in all four quadrants, with scattered retinal hemorrhages and a swollen, hemorrhagic optic disc. There was retinal whitening in the territory of cilioretinal artery (Figure 1). On clinical basis, she was diagnosed with combined central retinal vein and cilioretinal artery occlusion of right eye. Optical coherence tomography (OCT) showed macular edema with central macular thickness of $572 \mu \mathrm{m}$ in right eye and $222 \mu \mathrm{m}$ in left eye (Figure 2A and 2B). A low dose fluorescein angiography was done which confirmed delayed filling of the vein with blocked fluorescence due to hemorrhage and occluded cilioretinal artery (Figure 3 ). She was given goniomassage and underwent anterior chamber paracentesis and was started on eye drop dorzolamide hydrochloride $2 \%$ thrice a day. We decided to wait and observe. The patient was sent for investigations including full blood count, fasting blood sugar, erythrocyte sedimentation rate, renal, liver function tests, lipid profile and thrombophilic screening (serum homocysteine, serum fibrinogen, protein $\mathrm{C}$ and $\mathrm{S}$ levels, lupus anticoagulant), the results of which came out within normal range. The Carotid Doppler study was also normal. Patient was referred to cardiologist and gynecologist for if any intervention required.

After the intensive investigative workup and consultation with cardiologist and gynecologist, intervention in form of intravitreal steroid was suggested to the patient. The written informed consent was taken after explaining all risks and benefits to the patient. Following all aseptic precautions, intravitreal injection of triamcinolone acetonide ( $2 \mathrm{mg} / 0.05 \mathrm{ml}$ ) was given $4 \mathrm{~mm}$ behind the limbus in the inferotemporal quadrant in R/E. Four weeks after the treatment, visual acuity improved to $6 / 9$ in R/E. Slit lamp examination was normal with IOP of $17 \mathrm{mmHg}$ in both eyes. Fundus R/E revealed resolution of hemorrhages and artery occlusion area also showed less pallor (Figure 4A). OCT R/E also showed reduced mac-
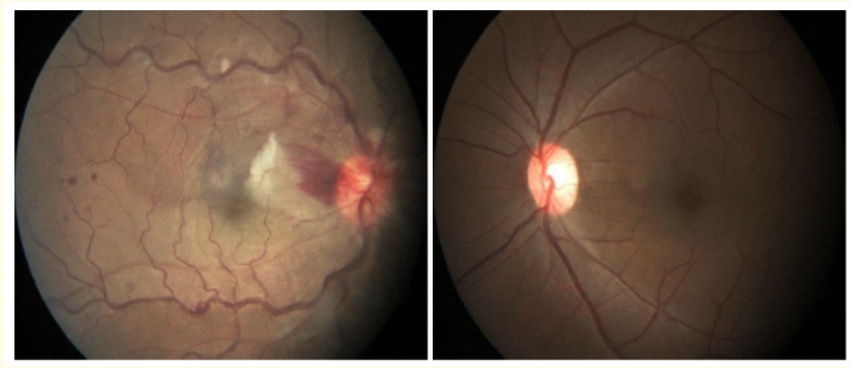

Figure 1: Fundus photo of both eyes at the time of presentation.
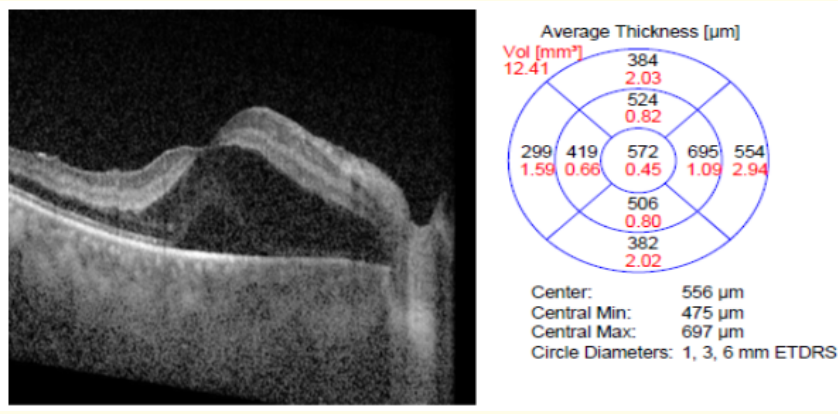

Figure 2A: OCT of right eye at time of presentation.
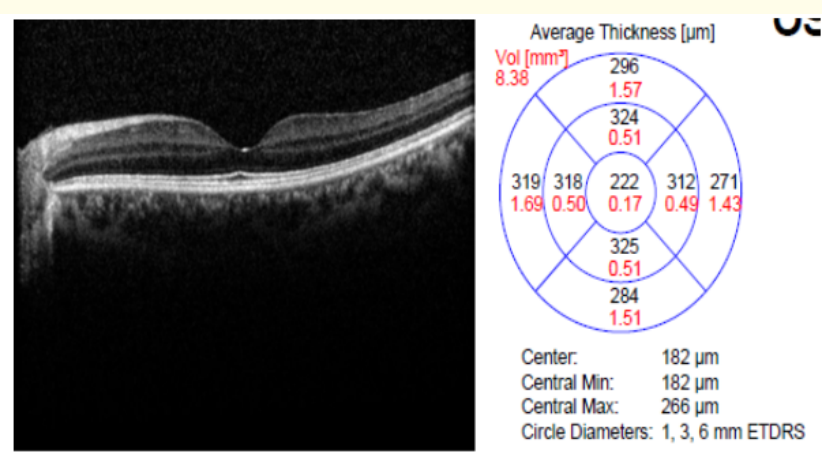

Figure 2B: OCT of left eye at time of presentation. 

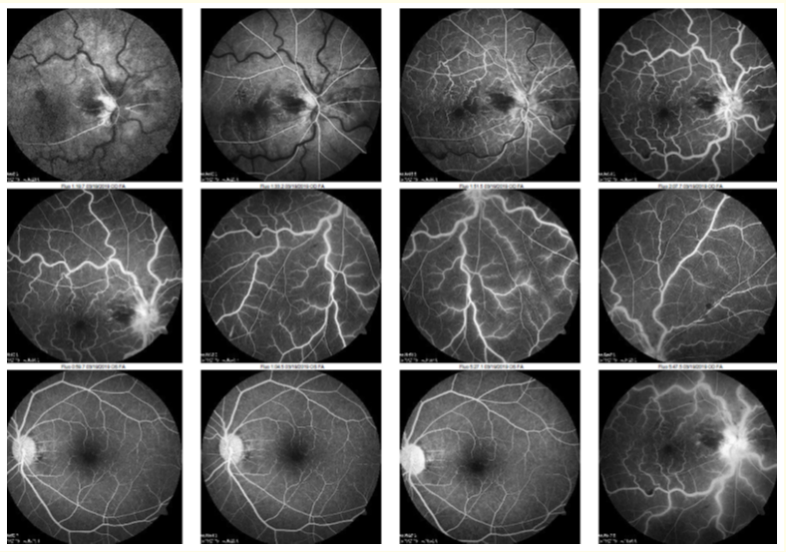

Figure 3: Fundus fluorescein angiography picture at presentation.

ular thickness of $217 \mu \mathrm{m}$ (Figure 4B). We followed up the patient on monthly basis and her vision remained stable with no rise in IOP. She gave birth to a healthy baby at full-term with no complications in pregnancy or parturition reported.
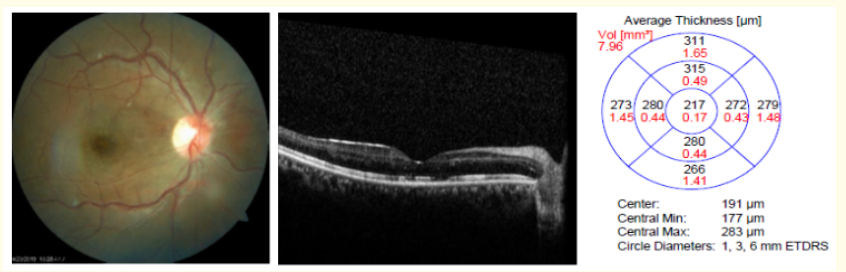

Figure 4: Fundus photo and OCT of right eye at 4 weeks post Intravitreal triamcinolone acetonide.

\section{Discussion}

The range of pregnancy associated-ocular changes is broad, one of which is retinal vascular occlusion. There have been reported cases of both retinal artery and vein occlusion [1-4]. In the reported cases, most of the patients had some systemic association like protein S deficiency [1], eclampsia [2], protein C deficiency [3] and diabetes mellitus [4]. The present case is also a young female with pregnancy as a predisposing factor. The thrombophilic screening however came normal in our case. Though the present patient do have a history of pre eclampsia in her previous pregnancy which has been reported as a cause of vascular occlusion in pregnancy [2].

The cause of visual loss in combined vascular occlusion can be both ischaemic retinal insult and macular edema. In the present case OCT recorded $572 \mu \mathrm{m}$ thickness. The various modalities of treatment available are anti vascular endothelial growth factor (VEGF) and steroids and laser. We did not consider an intravitreal injection of anti- VEGFs to treat because of possible risk of miscarriage or teratogenic effect on the fetus. Also, the anti VEGFs come under pregnancy category $C$ drugs and steroids under category $B$ drug indicating that steroids are safer than anti-VEGFs. Moreover, dexamethasone is known to be used in pregnancy in cases of preterm labour for fetal lung maturation and hence is safe. In literature, the use of dexamethasone and triamcinolone for treatment of macular edema in pregnant female have been reported $[5,6]$. Also, Degenring and Jonas., et al. reported that after an intravitreal injection of 20 to $25 \mathrm{mg}$ triamcinolone acetonide, its serum levels did not differ significantly between preoperatively ( $0 \mathrm{mcg} / \mathrm{L})$ and postoperatively $(0.065 \mathrm{mcg} / \mathrm{L})$ [7]. Thus indicating that intravitreal steroids can be a good treatment option in such cases in pregnancy. In the present case, the macular edema resolved effectively with no raise in IOP and visual acuity was maintained with no effect noted on baby and mother.

\section{Conclusion}

We propose that intravitreal triamcinolone injection may be a viable treatment modality for management of macular edema in pregnant patients but only after thorough investigative work up and consultation with an obstetrician.

\section{Conflict of Interest}

Authors does not have any financial interest or any conflict of interest whatsoever.

\section{Bibliography}

1. Will S K., et al. "Retinal artery and vein thrombotic occlusion during pregnancy: markers for familial thrombophilia and adverse pregnancy outcomes". Clinical Ophthalmology 10 (2016): 935-938.

2. Shilpa Y D., et al. "Bilateral central retinal artery occlusion in a case of eclampsia". Egyptian Retina Journal 5 (2018): 50-52. 
3. Ahmad Syed S., et al. "Protein S Deficiency Related Retinal Artery Occlusion in a Pregnant Chinese Woman". American Journal of Medical Case Reports 2.9 (2014): 175-180.

4. Loh MA., et al. "Bilateral central retinal vein occlusion in a pregnant lady". Medical Journal of Malaysia 72.2 (2017): 130-132.

5. Yoo R., et al. "Dexamethasone intravitreal implant for diabetic macular edema in a pregnant patient". International Journal of Ophthalmology-IJO 9.10 (2016): 1524-1527.

6. Fazelat A and Lashkari K. "Off-label use of intravitreal triamcinolone acetonide for diabetic macular edema in a pregnant patient". Clinical Ophthalmology 5 (2011): 439-441.

7. Degenring RF and Jonas JB. "Serum levels of triamcinolone acetonide after intravitreal injection". American Journal of Ophthalmology 137.6 (2004): 1142-1143.

Volume 4 Issue 9 September2021

(C) All rights are reserved by Minakshi Sheokand., et al. 